\title{
Experiencias
}

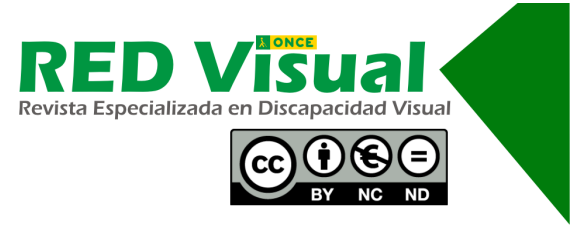

\section{Campus de Tecnología para alumnos con discapacidad visual estudiantes de Secundaria y Bachillerato}

Technology campus for secondary school students with visual disability

M. T. Corbella Roqueta, S. Boix Hernández, C. Mallo Robles, A. Gómez García, M. Cantón Soriano

\section{Resumen}

Este artículo muestra la experiencia de celebración del i Campus de Tecnología para alumnos con discapacidad visual, estudiantes de Secundaria y Bachillerato en España, en el Centro de Recursos Educativos de la ONCE en Barcelona. Con el objetivo inicial de despertar el interés científico y tecnológico de los alumnos en un área de especial dificultad, se han realizado diversas actividades en las áreas de mecánica, electricidad, robótica y proyectos finales, así como una mesa redonda con expertos afiliados que trabajan en tecnología. Se muestran las herramientas, recursos, adaptaciones y estrategias disponibles para el aprendizaje y práctica de estas materias. El campus se completó con dos visitas externas: CosmoCaixa y el centro de automoción Monlau en Barcelona, donde los alumnos pudieron completar su experiencia en el mundo de la tecnología.

\section{Palabras clave}

Discapacidad visual. Tecnología. Robótica. Mecánica. Electricidad.

\begin{abstract}
This article describes the First Technology Campus for Spanish secondary school students with visual disability, held at ONCE's educational resource centre in Barcelona. In keeping with the initial aim of sparking students' scientific and technological interest in an area of particular


difficulty, activities were organised around the fields of mechanics, electricity, robotics and end-of-course projects. The congress also featured a roundtable with ONCE members working as experts in technological endeavours. Participants were introduced to the tools, resources, adaptations and strategies available for learning and practising these professions. The activity was supplemented with two outings, to CosmoCaixa and the Monlau automotive facility in Barcelona, where students were further introduced to the world of technology.

\section{Key words}

Visual impairment. Technology. Robotics. Mechanics. Electricity.

\section{Introducción}

En la escuela del siglo XXI, el alumnado está inmerso en una cultura tecnológica global que le acompañará en todo su proceso de aprendizaje. Este aprendizaje requiere una atención específica en la adquisición de los conocimientos y destrezas que le serán necesarios para tomar decisiones y resolver los problemas que la sociedad le plantea. Por ello, debe aprender a tener la capacidad de actuar sobre el medio y mejorar la calidad de vida, manejándose en este entorno desde una visión crítica y formada.

En general, la tecnología es un área de especial dificultad para el alumnado con discapacidad visual, dado que la mayoría de los instrumentos, programas y procesos que se utilizan o se realizan no son especialmente accesibles. Por este motivo, se plantea la celebración del I Campus de Tecnología para alumnos con discapacidad visual en la ONCE.

Esta experiencia ha tomado como modelo otros campus que se celebran en la National Federation of the Blind, en EE. UU., en los que se promueve el interés de los alumnos con discapacidad visual por la ciencia, la tecnología, la ingeniería y las matemáticas.

\section{Objetivos y nivel de consecución}

Los objetivos del I Campus de Tecnología para alumnos afiliados a la ONCE de entre $1 .^{\circ}$ y $4 .^{\circ}$ de la ESO y Bachillerato con la asignatura de Tecnología, realizado en 
el Centro de Recursos Educativos (CRE) de la ONCE en Barcelona entre los días 25 y 27 de marzo de 2019 fueron los siguientes:

- Contribuir de manera práctica a la adquisición de una cultura tecnológica global que les permita comprender el mundo técnico que les rodea.

- Conocer la visión de profesionales afiliados a la ONCE expertos en el mundo de la tecnología con el objetivo de transmitirles su experiencia en este campo y contribuir a despertar su interés en el mundo tecnológico, no solo como usuarios sino como partícipes activos en la sociedad.

- Adquirir los conocimientos y destrezas necesarios para trabajar en el área de tecnología.

- Realizar actividades prácticas, habituales en los centros educativos y vinculadas al currículo escolar de su etapa educativa, accesibles y especialmente adaptadas en las áreas de electricidad, estructuras y mecanismos, y de programación y robótica.

- Dar a conocer las herramientas, recursos, adaptaciones y estrategias disponibles en el Seminario de Tecnología para el aprendizaje y práctica de estas materias.

- Trabajar en equipo, compartir inquietudes en el área de Tecnología, perdiendo el «miedo» a trabajar y optar por vocaciones científico-tecnológicas.

- Compartir experiencias y crear un grupo de amigos y compañeros para colaborar en el futuro.

- Dar a conocer cómo las actuales tecnologías nos permiten la conexión con profesionales a distancia (audio y videoconferencia) para compartir sus conocimientos.

Tras la celebración del Campus todos los miembros del Seminario de Tecnología participantes en la organización y realización del mismo valoraron muy positivamente su realización, así como se valoró como éxito el nivel de consecución de los objetivos planteados. 


\section{Metodología}

La metodología empleada en los talleres de mecánica, electricidad, robótica y proyecto final se ha basado en una didáctica activa, siguiendo los principios del constructivismo. Se ha partido del nivel de desarrollo del alumnado, considerando tanto sus capacidades y habilidades como sus conocimientos previos. El trabajo de los talleres en el CRE se realizó en pequeños grupos. Se trabajaron los mismos contenidos y actividades, pero adecuándolos al nivel académico del alumnado.

Otra metodología de trabajo utilizada en el taller de robótica es la del Aprendizaje Basado en Proyectos (ABP) que permite desarrollar el pensamiento crítico y mejorar las habilidades de resolución de problemas. Una vez planteado el reto, los alumnos debían colaborar entre ellos para programar el robot consiguiendo el objetivo planteado.

El objetivo de estas metodologías de trabajo ha permitido darle al alumno con discapacidad visual la oportunidad de desvelar sus capacidades y potencialidades, estimulando la motivación a través de actividades que permiten poner en práctica los conocimientos ya adquiridos en el aula ordinaria, y que, muchas veces por falta de accesibilidad, no han podido experimentar.

La distribución de los grupos de trabajo, por niveles educativos y con alumnos con resto visual y con ceguera indistintamente, ha permitido incorporar la metodología del Aprendizaje Cooperativo. De este modo, los alumnos han mejorado la atención y la adquisición de conocimientos, ya que trabajando conjuntamente han creado vínculos de amistad que han permitido realizar con éxito sus tareas apoyándose en el trabajo en equipo.

Siguiendo con el objetivo de convertir al estudiante con discapacidad visual en protagonista de su proceso de aprendizaje, se ha realizado una mesa redonda de expertos con discapacidad visual, profesionales en el ámbito tecnológico, como una metodología activa de aprendizaje que ha permitido a los alumnos la participación, exposición de sus puntos de vista y sus propios razonamientos en el Área de Tecnología, estableciendo un diálogo con personas con discapacidad visual que trabajan en este campo en la vida real.

La visita al centro de automoción Monlau fue un claro ejemplo de Aprendizaje Basado en Proyectos, ya que los alumnos pudieron adquirir conocimientos de mecánica partiendo de problemas concretos y reales. Además, pudieron desarrollar sus competencias de comunicación y colaboración con los alumnos del centro. Un ejemplo de la metodología

Corbella, M. T., Boix, S., Mallo, C., Gómez, A., y Cantón, M. (2020). Campus de Tecnología para alumnos con discapacidad visual de Secundaria y Bachillerato. RED Visual: Revista Especializada en Discapacidad Visual, 76, 149-166. https://doi.org/10.53094/GWGA1075. 
de aprendizaje mentoring, ya que los alumnos del centro de estudios Monlau fueron los encargados de transferir sus conocimientos y el aprendizaje a través de su experiencia a nuestros alumnos con discapacidad visual, estableciéndose una relación personal y de confianza entre mentor y alumno. Un aprendizaje enriquecedor para ambas partes.

En definitiva, en el I Campus de Tecnología para alumnos con discapacidad visual se han utilizado diversas metodologías educativas que se están implementando en las aulas ordinarias de la escuela del siglo XXI, demostrando que también son necesarias y que se pueden utilizar en el aprendizaje con alumnos con discapacidad visual siempre que estén adaptadas y sigan criterios de accesibilidad.

\section{Contenidos}

En el Campus de Tecnología, los alumnos afiliados a la ONCE han trabajado diversos contenidos del Área de Tecnología, tales como electricidad, estructuras y mecanismos, programación y robótica. También se han reforzado conceptos de física y mecánica del motor.

Los alumnos también han aprovechado para reforzar sus conocimientos de informática pudiendo tomar notas en el ordenador, principalmente en el taller de robótica y programación. Los contactos previos al campus con los alumnos también se han realizado vía correo electrónico para potenciar el uso de esta herramienta de comunicación.

En el Taller de Mecanismos se han trabajado los siguientes contenidos:

- Concepto de mecanismo.

- Transmisión de movimientos y fuerzas: palancas, poleas, poleas de transmisión, correa de transmisión, engranaje...

- Hélices, aspas...

En el Taller de Electricidad se han trabajado los siguientes contenidos:

- Concepto de electricidad. 
- Circuitos en serie y paralelo.

- Representación gráfica de circuitos.

- Construcción de circuitos básicos con kits básicos de electricidad y componentes reales utilizados en el aula ordinaria.

En el Taller de Robótica se han trabajado los siguientes contenidos:

- Leer y comprender el planteamiento de un reto.

- Planificar y escribir instrucciones para conseguir un objetivo.

- Verificar la eficacia de las instrucciones.

- Revisión y mejora del programa, si es necesario.

- Conocer el funcionamiento de los robots BeeBot, Escornabot y Milo de Lego WeDo 2.0.

- Construir el robot si es necesario (Lego WeDo 2.0, siguiendo instrucciones táctiles y descriptivas).

- Programar el robot para resolver el reto planteado.

En la visita al centro de automoción Monlau se han trabajado los siguientes contenidos educativos:

- Conocimiento de un taller de automoción.

- Secciones de Carrocería, Mecanizado, Mecánica, Electrónica y Motos.

- Qué se hace en cada una de estas secciones.

- Limpieza de piezas, pinturas, revestimientos...

- Procesos de soldadura, limado de superficies... 
- Maquinaria específica en el mecanizado de piezas.

- Partes mecánicas del coche: cambio de ruedas, frenos, caja de cambios...

- Partes eléctricas de un coche: cambio de una batería, cambio de luces, funcionamiento del motor de arranque.

- Motos: diferencias entre una moto automática, de trial o de carretera, partes y elementos de una moto, banco de pruebas...

- Conocimiento de herramientas específicas de cada sección.

- Aspectos de seguridad en el trabajo. EPI en cada una de las secciones.

En la visita a CosmoCaixa se han trabajado los siguientes contenidos educativos:

- Concepto de electricidad. Movimiento de protones y electrones.

- Experiencia del generador de Van de Graaff. Electricidad estática.

- Experiencia de lluvia de electrones.

- La jaula de Faraday.

- Efecto punta. Funcionamiento de un pararrayos.

- Viaje a la Luna. Momento histórico. Preparación del viaje (traje, planetas, órbitas, movimientos, alimentación, utensilios). El viaje (la nave, el lanzamiento). Llegada a la luna (herramientas, concepto de ingravidez).

\section{Actividades}

Estos contenidos se han trabajado en las siguientes actividades:

a. Mesa redonda y coloquio con alumnos y profesionales con experiencia en el ámbito de la tecnología. 
b. Taller de mecanismos.

c. Taller de electricidad.

d. Taller de robótica.

e. Proyecto final.

f. Visita al centro CosmoCaixa.

g. Visita al centro de automoción Monlau.

h. Actividades de ocio y tiempo libre: Taller de Basket Beat y Taller de piscina.

i. Exposición de proyectos, puesta en común y valoración final.

Para la realización de los talleres, los alumnos se dividieron en tres grupos por nivel educativo (4. ${ }^{\circ}$ de la ESO - Bachillerato, $1.0^{\circ}$ de la ESO - 2.0 de la ESO, $1 .{ }^{\circ}$ de la ESO). Cada grupo disponía del monitor del taller, un miembro del Seminario de Tecnología de Barcelona y un maestro voluntario de soporte.

Para las actividades externas, los alumnos se organizaron en subgrupos y también estuvieron acompañados de los miembros del Seminario de Tecnología y por monitores de los centros visitados. Estas visitas se prepararon previamente en el Centro de Recursos Educativos con un dosier adaptado con láminas en relieve explicando las actividades a realizar, con objetos y vídeos relacionados con las visitas.

\subsection{Mesa redonda de expertos en el Área de Tecnología y personas afiliadas a la ONCE}

Aparte de estas experiencias prácticas preparadas en el I Campus de Tecnología, se ha realizado una Mesa redonda con tres profesionales afiliados expertos en el mundo de la tecnología con el objetivo de transmitir a los alumnos su experiencia en este campo y contribuir a despertar su interés en este mundo tecnológico, no solo como usuarios, sino también como partícipes activos en esta sociedad. A los expertos seleccionados se les solicitó su colaboración presentando una mezcla de conocimientos tecnológicos y su experiencia personal con las siguientes preguntas de partida:

- ¿En qué consiste tu trabajo?

- ¿Qué estudios han sido necesarios? O cómo ha sido tu aprendizaje para llegar...

- ¿Qué problemas, dificultades u obstáculos has tenido que superar, si ha sido el caso? ¿Cómo se han solucionado? 
- ¿Qué cosas positivas destacarías de tu experiencia?

- ¿Qué recomendaciones les harías a los alumnos que quisieran iniciar este camino en sus estudios?

- ¿Cómo ves el futuro?

Los alumnos tuvieron la oportunidad de escuchar sus explicaciones y de intervenir haciéndoles llegar sus dudas, opiniones y comentarios de forma muy amena.

\subsection{Taller de mecanismos}

Los alumnos han estudiado diferentes proyectos del taller de tecnología relacionados con mecanismos.

Se ha ilustrado el tema con láminas fúser, con las que han podido entender las representaciones gráficas de los diferentes elementos con los que iban a trabajar (poleas, correa de transmisión, aspas...) y el vocabulario básico de los mecanismos.

Proyecto práctico: molino de aspas

Objetivos:

- Planificar y ejecutar un proyecto técnico utilizando materiales y herramientas correctamente.

- Conocer y experimentar mecanismos internos que logran transmitir y transformar el movimiento y la fuerza para conseguir el efecto apropiado.

- Utilizar herramientas de corte, limado, perforado, atornillado... de forma segura, teniendo en cuenta las adaptaciones de las técnicas para alumnos ciegos.

El desarrollo del proyecto se ha llevado a cabo de forma individualizada, de modo que cada alumno ha ido construyendo la estructura en la que se han montado los diferentes elementos (soportes, manivela, ejes...) para poder transmitir el movimiento por medio de poleas enlazadas por una correa hasta hacer girar las aspas del molino. 
Los objetivos del taller de mecanismos se pueden valorar como satisfactoriamente logrados, tanto para los alumnos que han experimentado el aprendizaje a través del desarrollo del proyecto, como para los profesionales que han intervenido en el diseño y apoyo.

\subsection{Taller de electricidad}

En este taller se han explicado las propiedades de la electricidad: conducción, voltaje, vatios, y medios aislantes. A continuación, han conocido el material con el que íbamos a trabajar, y se han hecho pruebas con las pilas, leds, cables y zumbadores. Después han realizado una práctica con la plastilina conductora. Han estudiado dos tipos de circuitos eléctricos: en serie y en paralelo. Una vez estudiados con láminas (tinta y fúser), los alumnos las han reproducido en papel. Por último, y antes de comenzar a trabajar los circuitos en los estuches de electricidad, se les han mostrado todos los componentes de los dos estuches.

Proyecto práctico: realización de los circuitos en serie y paralelo

Objetivos:

- Conocer los diferentes elementos sobre la electricidad y su funcionalidad.

- Saber conectar los diferentes elementos en la placa teniendo en cuenta el dibujo de los circuitos.

- Distinguir claramente los elementos en serie y los que están en paralelo.

- Detectar fallos en la conexión del circuito y saber solucionarlos.

- Aplicar medidas de seguridad utilizando las herramientas y procesos adecuados.

Cada alumno ha hecho sus propios circuitos y láminas. Todos disponían de las herramientas necesarias y un estuche de electricidad por alumno. Había dos modelos de estuche, uno más adecuado para los alumnos sin resto visual -ya que las conexiones eran con corchetes- y otro con cables con conexión de cocodrilo. Algunos alumnos, al terminar de hacer los circuitos, improvisaron otros con elementos del estuche. 
La valoración del taller es muy positiva, ya que la mayoría aprendieron cosas que antes no habían experimentado, y fue muy bien el contar con maestros de apoyo en los diferentes talleres para hacer una atención más individualizada.

\subsection{Taller de robótica y programación}

Se realizaron tres prácticas diferenciadas:

- En la primera se han explicado los conceptos de robótica unplugged y se han trabajado los conceptos básicos de programación con el BeeBot y un tablero adaptado de $3 \times 3$. Una vez planteado un reto a los alumnos, debían programar en el robot, pulsando botones, el movimiento del robot hasta llegar a su objetivo en el tablero. Por ejemplo, llegar a una casilla para recoger un objeto con el mínimo número de instrucciones al robot.

- En la segunda práctica se ha utilizado el robot Escornabot, un robot de «tecnología Maker», que además de con una botonera se puede programar con una aplicación móvil accesible. Este robot incorpora giros de $45^{\circ}$ y se puede programar con Arduino. Con este robot se incorporó el concepto de aprendizaje basado en el pensamiento (Thinking Based Learning). Los alumnos debían convertir la información recibida en conocimiento. Primero, pensaban y diseñaban su reto, analizaban la situación y, posteriormente, programaban el robot para conseguir su objetivo.

- En la tercera práctica los alumnos debían construir y programar un robot de Lego (Milo WeDo 2.0). Siguiendo unas instrucciones visuales/textuales en el ordenador y con el soporte de láminas en relieve, construían en equipo el robot Milo. Seguidamente, realizaban la programación con bloques adaptados en relieve $y$, en colaboración con el compañero con resto visual o el profesor, las traspasaban al software de Lego de la tablet. Observaban el resultado de la programación y verificaban el cumplimiento de su reto.

\section{a) Proyecto final: coche con motor}

La propuesta de trabajo de este proyecto final pretendía englobar los conceptos trabajados en los distintos talleres y tratar de reproducir los proyectos de trabajo que en la materia de Tecnología se realizan en las aulas ordinarias. 
Para ello, a partir de todos los materiales (palos de polo, palillos, micro-motor, engranajes, pila, interruptor, cable eléctrico, etc.), y con las adaptaciones necesarias, los alumnos construyeron un mini coche con motor.

Este proyecto también potenció la colaboración y el trabajo en equipo de los alumnos.

\section{b) Visita a CosmoCaixa, «Tintín y la Luna» y taller «Vive la electricidad»}

En la visita a CosmoCaixa los alumnos se dividieron en dos subgrupos. Todos los grupos realizaron las mismas actividades. En primer lugar, un grupo estuvo en el taller de electricidad mientras el otro estaba en la exposición «Tintín y la Luna». Cada grupo iba acompañado de un monitor de CosmoCaixa, y para la exposición dos monitores (uno recibía la explicación de «Tintín y la Luna» mientras el otro grupo realizaba la actividad de ingravidez). En la experiencia de ingravidez los alumnos estaban colgados mediante unos arneses a una grúa giratoria adaptada a su peso lunar para poder vivir la sensación de ingravidez.

La visita estuvo totalmente adaptada. Desde el seminario de Tecnología se preparó un dosier con las láminas en relieve de los experimentos del taller de electricidad y de la exposición de «Tintín y la Luna» y objetos para acompañar el relato de la exposición (partes de la nave en 3D, comida envasada, objetos que acompañaron a los astronautas, etc.). Previamente, también se trabajó la visita con los alumnos con un vídeo explicativo del viaje a la Luna realizado por un especialista del Seminario de Tecnología utilizando la herramienta de videoconferencia Zoom.

\section{c) Visita al Centro de Estudios de Automoción Monlau}

El grupo de alumnos se dividió en 5 subgrupos, los cuales, acompañados por dos profesionales del Seminario de Tecnología y voluntarios del CRE de Barcelona, recorrieron las distintas secciones del Centro de Estudios de Automoción Monlau.

En cada sección (carrocería, mecanizado, mecánica, electrónica y motos) fuimos atendidos por el responsable de la sección y por un grupo de alumnos de $2 .^{\circ}$ Ciclo Formativo de Automoción, que se encargó de mentorizar a nuestros alumnos.

Además de recibir las explicaciones teóricas correspondientes, los alumnos en cada sección realizaron actividades prácticas con sus alumnos mentores: pulir la carrocería 
de un coche; tocar las partes de un coche, del motor, de una caja de cambios; utilizar la pistola para cambiar la rueda de un coche; realizar el cambio de una batería de coche; tocar la maquinaria para el mecanizado de piezas; subir a una moto en el banco de pruebas; dar gas, etc.

Los alumnos vivieron toda la experiencia de un taller de automoción.

\section{d) Actividades de ocio y tiempo libre}

Las actividades lúdicas de Basket Beat y de natación contribuyeron a la cohesión del grupo y a fortalecer su responsabilidad y autonomía personal.

\section{e) Actividad de valoración final}

En esta actividad, los alumnos reflexionaron y dieron su opinión, en primer lugar, sobre algunas cuestiones preparadas previamente por el Seminario de Tecnología, dirigidas a conocer cuál era su experiencia en el Área de Tecnología en su ámbito y entorno escolar, y, posteriormente, realizamos una valoración específica del I Campus de Tecnología.

Aprovechamos también para recoger mensajes de agradecimiento para los alumnos del Centro de Estudios Monlau.

Área de Tecnología:

1. ¿Qué temas de toda la asignatura de Tecnología de tu instituto o colegio te gustan más?

2. ¿En qué temas de la asignatura has tenido más dificultades en tu instituto o colegio? ¿Cuáles?

3. ¿Has desarrollado proyectos de tecnología en tu instituto o colegio? De ser así, ¿podrías indicar en qué han consistido o qué se ha trabajado en ellos?

4. En los trabajos en grupo, ¿participas en las tareas de los proyectos de forma activa o colaboras con el grupo dejando las tareas manipulativas al resto mientras realizas partes más accesibles? 
5. ¿En ocasiones te han encargado trabajos diferentes a los del resto de los compañeros? ¿Recuerdas algún caso?

Sobre el Campus de Tecnología:

1. ¿Qué es lo que más te ha gustado del Campus?

2. ¿Puedes resumir brevemente qué has aprendido?

3. ¿A qué parte hubieras dedicado más tiempo?

4. ¿Se te ocurre alguna mejora para futuras convocatorias?

5. ¿Te volverías a presentar a otra convocatoria de un campus de tecnología?

\section{Valoración general de resultados}

La valoración de los asistentes al campus es muy positiva. Todas las actividades diseñadas les han gustado y han sido muy interesantes, aportándoles conocimientos y experiencias que habitualmente no encuentran en las aulas del instituto. Con sus palabras: «He aprendido muchas cosas».

En general, dada la temática del campus, se lo habían imaginado -reproduciendo sus palabras- «más teórico, más "pesado" y más dedicado a la informática», por lo que les ha sorprendido el formato práctico de todas las actividades.

En relación a las ponencias de la mesa redonda, las valoran de forma muy positiva. Con sus palabras: «Ver a gente que lo ha hecho antes anima», aunque proponen añadir modelos de alumnos de la universidad o de estudios superiores.

Las salidas al exterior también les han gustado mucho, destacando el contacto con los alumnos del Centro de Estudios Monlau, por mostrarles actividades y vivencias que difícilmente les enseñan en el instituto, y por el nivel de implicación, paciencia y de compromiso que presentaron con ellos.

Ante la pregunta sobre qué es lo que más te ha gustado, evidentemente, la mayoría piensa que la experiencia de subir a la moto, cambiar la rueda, aunque también mencionan la experiencia de ingravidez en el CosmoCaixa y poder sentir la sensación de la electricidad del taller «Vive la electricidad». 
Valoran el tiempo de las sesiones de talleres como corto, ya que en algunos casos les habría interesado disponer de más tiempo para poder finalizar los proyectos. En este sentido, algunos apuntan la idea de alargar los días de campus para disponer de más tiempo en las actividades.

Otro aspecto que valoran muy positivamente ha sido el poder estar con los compañeros y el buen grupo humano que se formó. En este sentido, también proponen disponer de más tiempo libre para poder convivir en el grupo.

En relación a propuestas para un próximo campus de tecnología, algunos de ellos añadirían algunos temas de informática, ocio accesible (juegos tecnológicos), etc.

En relación a la valoración de los miembros del Seminario de Tecnología y los organizadores de todas las actividades, nos gustaría destacar los siguientes aspectos:

- En conjunto, la experiencia ha sido altamente positiva y satisfactoria. Nos ha permitido generalizar y poner en práctica con un grupo de alumnos todas aquellas ideas, adaptaciones, estrategias y metodologías que muchas veces han sido diseñadas para situaciones específicas. El trabajo realizado ha servido para el aprendizaje no solo de los alumnos sino también del grupo del Seminario de Tecnología.

- El grupo de alumnos ha sido muy heterogéneo y, en muchos casos, no disponíamos de información respecto a las competencias/habilidades que poseían los alumnos. Aun así, los trabajos y actividades planificados se han adaptado a sus necesidades, y los colaboradores de los talleres han ayudado a aquellos alumnos que precisaban más atención. Seguramente, en futuras convocatorias estaría bien disponer de más información de los alumnos para poder ajustar tiempos, nivel de actividades, etc.

- Aparte de trabajar los contenidos específicos del Campus de Tecnología hemos tenido ocasión de observar las destrezas básicas de los alumnos en aspectos manipulativos, trazo de líneas y figuras utilizando la goma de dibujo, realización de composiciones espaciales, uso de herramientas básicas de taller, interpretación de planos y esquemas en relieve... En esta observación vemos grandes diferencias entre los alumnos y la necesidad de trabajar estos aspectos en el día a día. 
- Las salidas y actividades externas han aportado motivación y cohesión de grupo, y les ha permitido vivir experiencias novedosas.

- Como aspectos de mejora, y dada la creciente importancia del área tecnológica, en el futuro creemos que, para poder hacer llegar la experiencia a un máximo número de alumnos, debería repetirse periódicamente la celebración de este campus de tecnología, aunque sería conveniente poder realizar estos campus en unas fechas que no interrumpan el curso escolar. facilitando así el acceso a todos los alumnos.

M. Teresa Corbella Roqueta. Profesora. Coordinadora del Servicio TIC. Grupo Accedo. Centro de Recursos Educativos de la ONCE en Barcelona. Gran Vía de les Corts Catalanes, 394; 08015 Barcelona (España). Correo electrónico: mtcr@once.es.

Silvia Boix Hernández. Instructora en Tiflotecnología y Braille. Grupo Accedo. Centro de Recursos Educativos de la ONCE en Barcelona. Gran Vía de les Corts Catalanes, 394; 08015 Barcelona (España). Correo electrónico: sbh@once.es.

Carlos Mallo Robles. Profesor. Coordinador del Servicio TIC. Grupo Accedo. Centro de Recursos Educativos de la ONCE en Madrid. Avda. del Doctor García Tapia, 210; 28030 Madrid (España). Correo electrónico: cmro@once.es.

Agapito Gómez García. Maestro. Grupo Accedo. Centro de Recursos Educativos de la ONCE en Sevilla. Calle Campo de los Mártires, 10; 41018 Sevilla (España).Correo electrónico: aggg@once.es.

Mariano Cantón Soriano. Maestro. Centro de Recursos Educativos de la ONCE en Alicante. Avda. de Denia, 171; 03559 Santa Faz (Alicante, España).Correo electrónico: mcsor@once.es. 


\section{Apéndice \\ Reportaje sobre el Campus}

Figura 1. Póster del I Campus de Tecnología

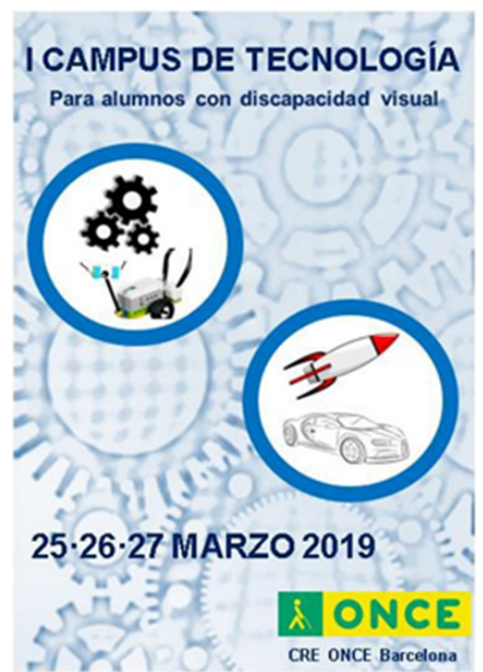

Figura 3. Taller de coche con motor

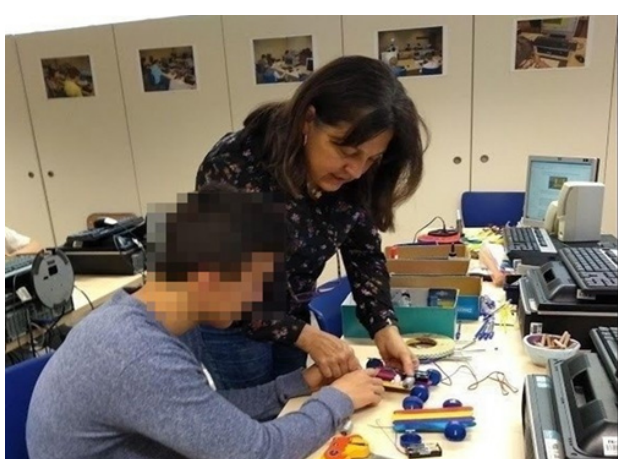

Figura 5. Taller de mecánica

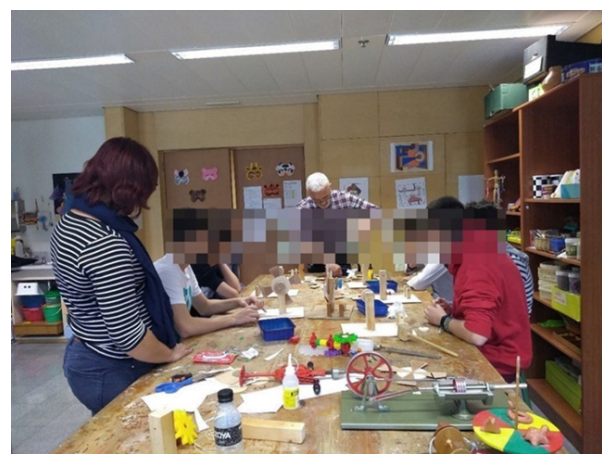

Figura 2. Pin identificativo de los alumnos

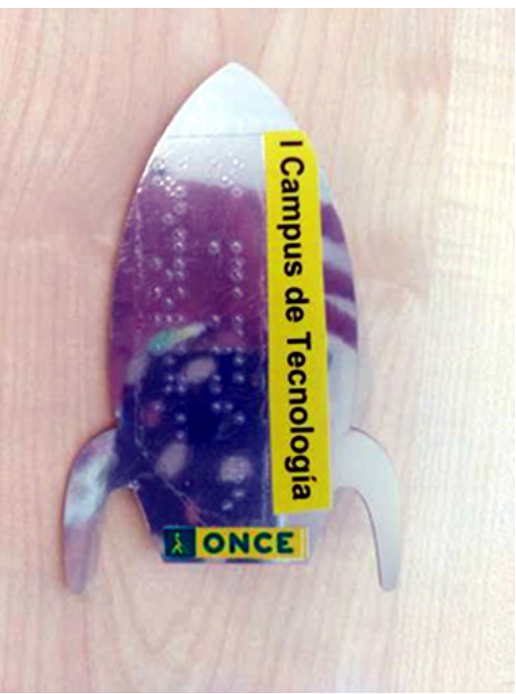

Figura 4. Taller de electricidad

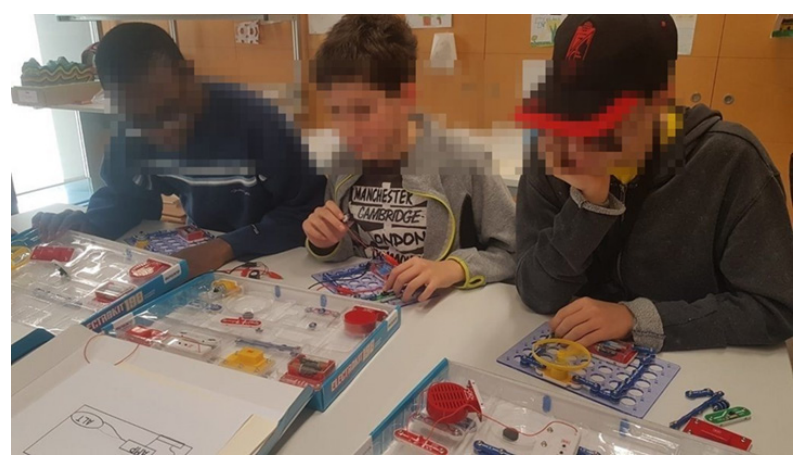

Figura 6. Taller de robótica

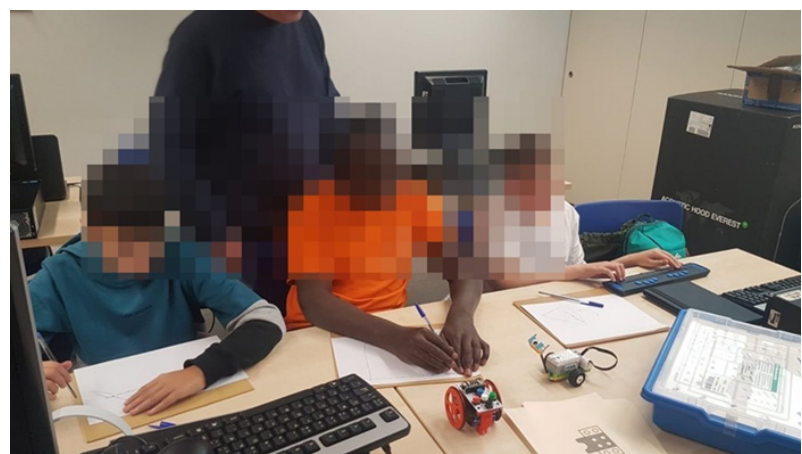

Corbella, M. T., Boix, S., Mallo, C., Gómez, A., y Cantón, M. (2020). Campus de Tecnología para alumnos con discapacidad visual de Secundaria y Bachillerato. RED Visual: Revista Especializada en Discapacidad Visual, 76, 149-166. https://doi.org/10.53094/GWGA1075. 
- RED Visual:: Revista Especializada en Discapacidad Visual "

$\cdot$ N.॰ 76 - Noviembre 2020 - ISSN 2660-4485 .

Figura 7. Taller de automoción

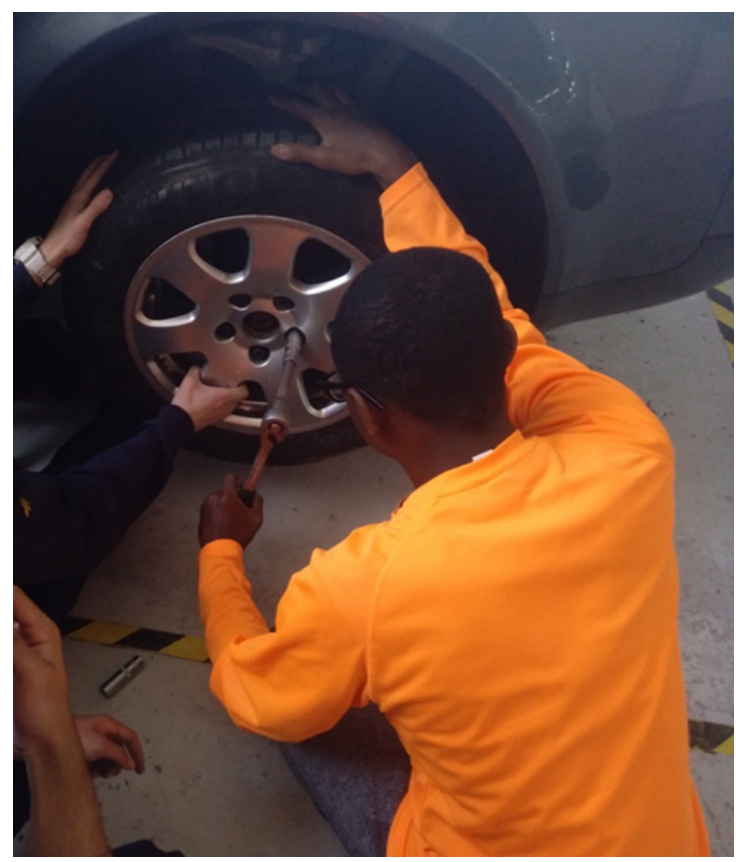

Figura 8. Taller mecánico

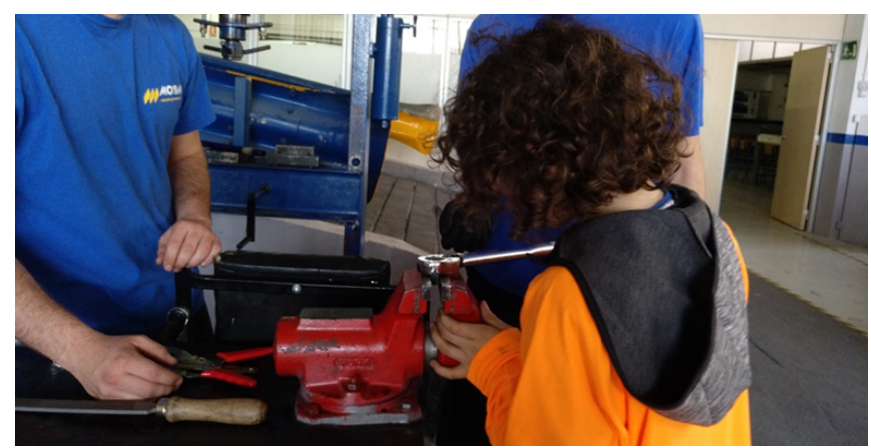

Figura 9. Taller de motos

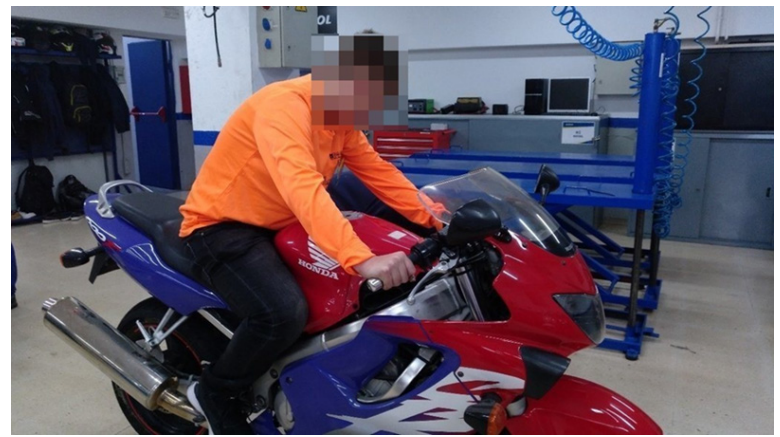

Corbella, M. T., Boix, S., Mallo, C., Gómez, A., y Cantón, M. (2020). Campus de Tecnología para alumnos con discapacidad visual de Secundaria y Bachillerato. RED Visual: Revista Especializada en Discapacidad Visual, 76, 149-166. https://doi.org/10.53094/GWGA1075. 